\title{
Rehabilitation Centred Design
}

\author{
First Author \\ AuthorCo, Inc. \\ 123 Author Ave. \\ Authortown, PA 54321 USA \\ author1@anotherco.com \\ Second Author \\ VP, Authoring \\ Authorship Holdings, Ltd. \\ Authors Square \\ Authorfordshire, UK AU1 2JD \\ author2@author.ac.uk \\ Third Author \\ AnotherCo, Inc. \\ 123 Another Ave. \\ Anothertown, PA 54321 USA \\ author3@anotherco.com

\section{Fourth Author} \\ YetAnotherCo, Inc. \\ 123 YetAnother Ave. \\ YetAnothertown, PA 54321 USA \\ author4@yetanotherco.com \\ Fifth Author \\ AuthorCo, Inc. \\ 123 Author Ave. \\ Authortown, PA 54321 USA \\ author5@anotherco.com \\ Sixth Author \\ AnotherCo, Inc. \\ 123 Another Ave. \\ Anothertown, PA 54321 USA \\ author6@anotherco.com

\section{Seventh Author} \\ AnotherCo, Inc. \\ 123 Another Ave. \\ Anothertown, PA 54321 USA \\ author7@anotherco.com

\section{Eighth Author} \\ YetAnotherCo, Inc. \\ A author8@yetanotherco.com
}

Copyright is held by the author/owner(s).

CHI 2009, April 4 - 9, 2009, Boston, MA, USA

ACM 978-1-60558-246-7/09/04.

\begin{abstract}
Stroke is a significant cause of disability, and is predicted to become a greater burden as population demographics shift. Research suggests that the completion of rehabilitation exercises can considerably improve function in damaged limbs, yet these exercises can be both boring and frustrating for patients to complete at home. New technologies create possibilities to support rehabilitation in motivating and entertaining ways, and, in this paper, we present a case study that illustrates the work of designing such technologies for a single user. Participation in this case study has highlighted some interesting tensions between designing for rehabilitation and designing for the user, and we provide a short discussion of these issues.
\end{abstract}

\section{Keywords}

Rehabilitation technologies, stroke, rehabilitation centred design

\section{ACM Classification Keywords}

H5.m. Information interfaces and presentation (e.g., $\mathrm{HCI}$ ): Miscellaneous.

\section{Introduction}

Stroke may be one of the biggest burdens to health and social care in the coming decades [3]. It is the leading cause of severe adult disability [2], limiting physical activity and affecting independence and quality of life. Evidence suggests that post-stroke rehabilitation exercises, if repeated often, and with sufficient quality 
of movement, can lead to increased mobility. However, individuals do not always practice exercises as prescribed by healthcare professionals. Sometimes these exercises may be practiced incorrectly, and sometimes the repetitive, monotonous and frustrating nature of the exercises may mean patients are not well motivated to complete these exercises. New sensing technologies open up possibilities for designing technology to help motivate and support rehabilitation in the home, and this is an area in which the <anonymous> project, a collaboration between physiotherapists, computer scientists, interaction designers, and engineers, aims to contribute. Through a user-centred approach that included interviews and workshops the long-term aim of this project became to assemble a rehabilitation toolkit that would facilitate the continuation of rehabilitation in the home environment. Our aim is to create such a technology through a "plug and play" approach, which might then allow a physiotherapist, patient or carer to match appropriate input devices with interesting content. To develop this toolkit, we have engaged in a user-centred manner, with four stroke patients, developing specifically tailored applications that can be used at home to support their rehabilitation, as well as to fit with their lifestyles, interests and motivations. We then hope to generalise from these four cases studies, towards the development of a toolkit that is relevant to the wider community of individuals who are living with stroke. In this paper, we present one of our four case studies constructed around an individual with a damaged left hand. The most significant contribution of our case study is an illustration of some of the competing and complicated needs of participants who have had strokes, and we hope that this will assist other designers working with rehabilitation technology.

\section{Illustrative Case Study}

Solomon (not his real name) is in his fifties and lives with his partner. Initially Solomon's stroke limited the whole of the left hand side of his body, causing difficulties with walking and with functional use of his left hand and arm. However, one year later Solomon has regained substantial amounts of movement in his left leg, and his left arm is now capable of gross movements, with limited fine motor control in his left hand. Since having the stroke, Solomon notes that it takes him much longer to think through complex ideas, and he is no longer able to partake in activities he once enjoyed, like playing the stock market. In terms of aims for recovery, Solomon felt that it was the lack of fine control in the fingers of his left hand that was his main source of problems, and, in particular, his ability to release objects. One of the earliest stages of our design work with Solomon was the identification, with the help of a physiotherapist, of an exercise that could improve this function. We settled on attempting to improve Solomon's ability to move his thumb away from the fingers in a controlled manner.

In our first design meeting with Solomon, conducted in his home by an interaction designer and a physiotherapist, we found that Solomon himself was very unsure as to the kind of content which could motivate him to rehabilitate his arm at home. However, through our conversations with Solomon we gleaned that he liked watching horse racing and playing chess. After this design meeting, we then developed and sketched out a number of potential designs for systems, which were described to him at a second design meeting. These included a "bet-against-themachine" game, a horse racing game and a chess game. Solomon's eyes lit up upon hearing that we 


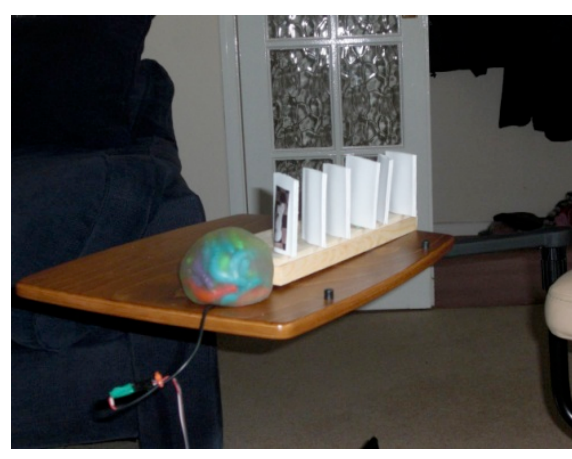

Figure 1: Tangible chess board

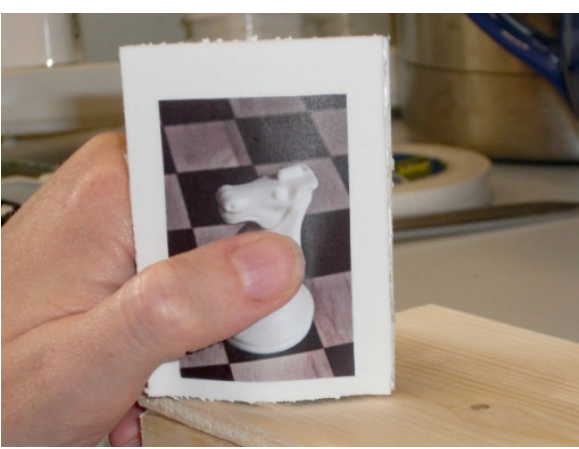

Figure 2: Grasping a chess piece could develop a technology so that he could play chess and exercise his hand and arm at the same time.

Having chosen to develop a rehabilitative chess game, we took a rough prototype of an input device that would enable Solomon to complete rehabilitation exercises in the home, whilst playing chess (figure 1 ). This consisted of six thin cards, each containing a pressure sensor, and each representing one of the six categories of piece in a chess game (pawn, bishop, knight, rook, queen and king), as indicated by an image placed on the card (figure 2). Our aim was to be able to interface this device to a computer chess game, in such a way so that, for Solomon to make a move, he must first open out his fingers from his thumb in order to "grasp" the piece that he wants to play with. This grasp indicates to the chess game that Solomon has chosen to play, for example, a pawn in his next turn. Solomon could then use his right hand to control a mouse and to interact with the game in a normal way (see figure 3), i.e. by choosing a specific piece, and by indicating to where it should move. In addition, our device featured a soft pad, to the left of the board, which could provide a resting space for Solomon's hand, and which allowed Solomon to pause the game.

Solomon seemed pleased with the design of the chessboard, even commenting that he could imagine taking this technology on holiday with him. However, in watching Solomon interact with the chessboard it was evident that adaptations would need to be made to ensure Solomon's game play was not too infringed upon by exercise and fatigue. For example, the meeting illustrated how difficult and tiring even making only a couple of moves would be for Solomon. Together we adapted the protoype such that at the start of each chess game Solomon would choose two categories of piece to exercise with by placing the representations of those pieces onto the tangible chessboard. The remaining four categories of pieces would then be controlled solely through a mouse click, or keyboard entry. Solomon seemed happy with this design change, and the chess interface is currently under development.

\section{Discussion}

There are a number of tensions evident in our design process. Perhaps at the forefront of these is the need to find the right balance between deploying an efficient and easy interaction for Solomon to play chess through, versus designing an interaction that supports rehabilitation and motivation. For example, a traditional user-centred approach, focused on ease and efficiency of use may have seen us providing a simple pressure sensor clicker device for use in Solomon's left hand to act as a mouse click. An interaction using this technology may have seen Solomon using his right hand to hover a mouse arrow over the chess piece that he wishes to play and squeezing the clicker between his thumb and fingers of his left hand to select the piece. Solomon would then move the mouse arrow again with his right hand to the square where he wants to move the piece, and again squeeze the clicker with his left hand to indicate the move. Such a device would meet traditional usability requirements, with the interaction being efficient, easy to learn and reasonably satisfying to the user. However, whilst such an interaction meets some rehabilitation needs (Solomon would be completing exercise with his left hand) it falls short of providing an interaction that feels integral to the game as well as providing an action for his left hand that is meaningful and functional. These features of an interaction are important for both physiotherapy and 


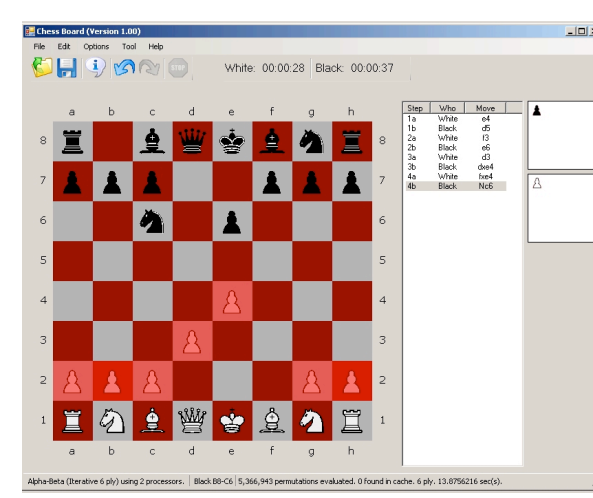

Figure 3: A chess game in progress in which the Solomon is controlling pawns with the sensor input device. He is unable to select any pawns without first squeezing the card representing the pawn. This software has been build upon open source chess software supplied by code project: <anonymous> motivational requirements. In terms of physiotherapy, functional and meaningful movements have been shown to positively influence motor learning [1]. Additionally, motivation theory [4], would suggest that feelings of growing competence are integral to the development of intrinsic motivation. Relegating the left hand to complete the menial task of a mouse click may instead perpetuate feelings of incompetence in the hand. Instead our tangible interface creates a hand movement designed to feel both integral in function and meaning to the game play output. It is the work involved in doing this that creates a second tension. A plug and play approach implies being able to pick an input device off the shelf and simply plug it into an appropriate output device. What we have found however is that there is significant design work entailed in appropriating an input device such that it feels functional and meaningful with respect to the chosen output. A third tension in the design process surrounds the incorporation of exercise into a participant's hobby. Solomon finds playing chess a pleasurable and enjoyable activity and we have opted to incorporate his rehabilitation exercises into this game with the hope of increasing Solomon's motivation to complete these exercises. We tried to balance Solomon's rehabilitation needs with the Solomon's chess game needs through designing an interaction that requires Solomon to only exercise when playing just two categories of pieces. However, this system is yet to be deployed and as such we do not know what the real impact of such merging of activities will be. Completing this design process has illustrated that rehabilitation centred design can be at odds with user centred design and more generally usability principles. Whilst we have worked with Solomon to create something that meets his needs, we are in fact creating a technology that may induce frustration and potentially negatively impact the chess game play. The user continues to be at the centre of our design cycle, yet the emphasis on the user is significantly more multi-faceted with competing and complex demands around physiotherapy, motivation and technical needs. Whilst this case study has explored the tensions around designing technologies to motivate stroke rehabilitation, we believe this need to balance the multi-faceted needs and wants of a user may be reflected in the design of technologies that care for the elderly, or even the design of games.

\section{Future Work}

Over the next few months the chess game prototype will be deployed in Solomon's home for the period of one month. This deployment will enable us to explore the extent to which the chess game motivates Solomon to complete his rehabilitation exercises at home.

\section{Acknowledgements}

Many thanks to our four participants without whom this work could not occur.

\section{References}

[1] Ma, H., C. Trombly, and C. Robinson-Podolski, The Effect of Context on Skill Acquisition and Transfer. AJOT, 1999. 53(2): p. 138-144.

[2] Millan, M. and A. Davalos, The Need for New Therapies for Acute Ischemic Stroke. Cerebrovasc Dis 2006. 22 (Suppl 1): p. 3-9.

[3] Pridmore, T., et al., Mixed Reality Environments in Stroke Rehabilitation: Interfaces across the Real/Virtua Divide Int J Disabil Hum Dev, 2007. 6(1): p. 3-10.

[4] Ryan, R. and E. Deci, Self-Determination Theory and the Facilitation of Intrinsic Motivation, Social Development, and Well Being. American Psychologist, 2000. 55(1): p. 68-78. 